\title{
Smart City by Smart Lighting: Utilizing Smart Lighting in Urban Texture Based on Effective Using of Power to Save in Macro Economy and Create Diversity in Cities
}

\author{
Amir Reza Goudarzi* \\ Department of Architecture, Islamic Azad University, Iran
}

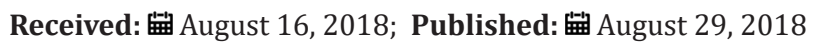

*Corresponding author: Amir Reza Goudarzi, Department of Architecture, Engineering faculty, Arak branch, Islamic Azad University, Arak, Iran

\begin{abstract}
Lighting is the requirement of the present world realized by artificial lighting especially at nights. However, the price paid is not as much rational threatening the national economy. Today, permanent facing with economic problems requires utilizing any potential for economic growth. Smart lighting prevents energy and capital losses; it is applied whenever it needed; and smart streetlights provide lighting whenever any human or vehicle passes. Thus, not only the two factors of lighting and saving national capitals will always last at negligible error, but also, diversity and aesthetic elements granted to cities enjoying the social and economic convenience. It assumed that using smart lighting is one condition of providing these conveniences. However, the question raised here is the infrastructures and utilizing equipment, particularly in traditional cities, to be responded. In conclusion, utilizing such system can lead to cost return and saving in order to achieve national objectives and to unconsciously teach aesthetic, change, and saving culture.
\end{abstract}

Keywords: Smart Lighting; Saving; Economy; Capita; System

\section{Introduction}

Light has always been the core factor of applying the supreme sense namely visual sense. The light encompasses the beautiful, great world and introduces the existence identity; all living organisms relates to this light. Today, visual sense is the most critical human sense. Human being obtains environmental information through using its visual sense Ghotbi Ravandi et al. [1]. Technology growth introduced artificial lighting to human life to facilitate nightlife like day and to avoid limiting working hour, entertainment, and traffic to daylight through utilizing the overall 24 hours. However, in addition to its advantages, some problems and disadvantages may emerge including excessive costs of urban lighting at nights that is in contrast with economic saving. Large, unhelpful costs spend on such lightings rather than developing and evolution of a community. For instance, when there is no human and vehicle traffic in street at night, it is not necessary to have streetlights on; and or, avoid overuse by reducing the brightness; and the brightness increases in terms of the number and volume of passing vehicles or people and reaches maximum level, if needed.
Several implementing examples and writings can be seen in this domain, particularly in developed countries. Walt Patterson, in its book "Keeping the lights on", provided useful information on night lighting and lighting technologies; however, he did not directly address smart lighting. Jason Makansi, in its book "Lights out", indicated some expressions on electricity and smart lighting. Catherine Rich in "Ecological Consequences of Artificial Night Lighting" studied lighting and its psychological effect at night by using smart system. Moreover, Benjamin Crowell, in its book "Electricity and Magnetism", utilized innovative electricity systems in smart lighting and helped better lighting system technology. Fred Schubert, in a paper, used this system and described obtaining such system. However, the best example is "Smart Light Responsive Materials Azobenzene-Containing Polymers and Liquid Crystals" that comprehensively discussed this area. Further, Hideaki NII paper opened a new, different approach in smart lighting in which the secret of adjusted smart lighting can be found out.

Ahmadiyan [2], in "An Introductory to Lighting Designing Principles", referred lighting basics, optical equipment, and 
lighting estimations in urban buildings and spaces. The major objective is economic, social, and cultural convenience so that add functionalism to the city face in addition to diversity and beauty through developing smart lighting knowledge. It also helps macro economy by the aid of this technology and avoids wasting national capitals and energies. The human being must try in achieving its next generation's ideals and pay for basic, reparable costs to attain the outcomes in long-term. Regarding the theoretical demonstrated assumptions, optimally using of smart lighting technology donates comfort and convenience to human life including economic comfort. However, this question raises that what are the necessary implementing infrastructures? or is this system functional in targeted lighting in traditional cities?

\section{Research Methodology}

In this study, descriptive-analytical (quantitative and qualitative) research method was used as survey and library method. The study continued by reviewing studies and their advantages and disadvantages. We tried our best to cover all research aspects so that the best results could be obtained. In addition, we studied a few books on subjects in relevant fields to be able to interpret the results accurately.

\section{Explanations}

As is evident in Figure 1 excessive lighting is associated with the wealthy countries of the world, places where people can afford to consume energy to illuminate the environment all night. The developing world, although supporting much higher population densities, is shown to be much darker at night, with fires used as lights rather than electric fixtures. Even in the developing world, however, industrial resource extraction is associated with artificial lighting Elvidge and ML Imhoff [3]

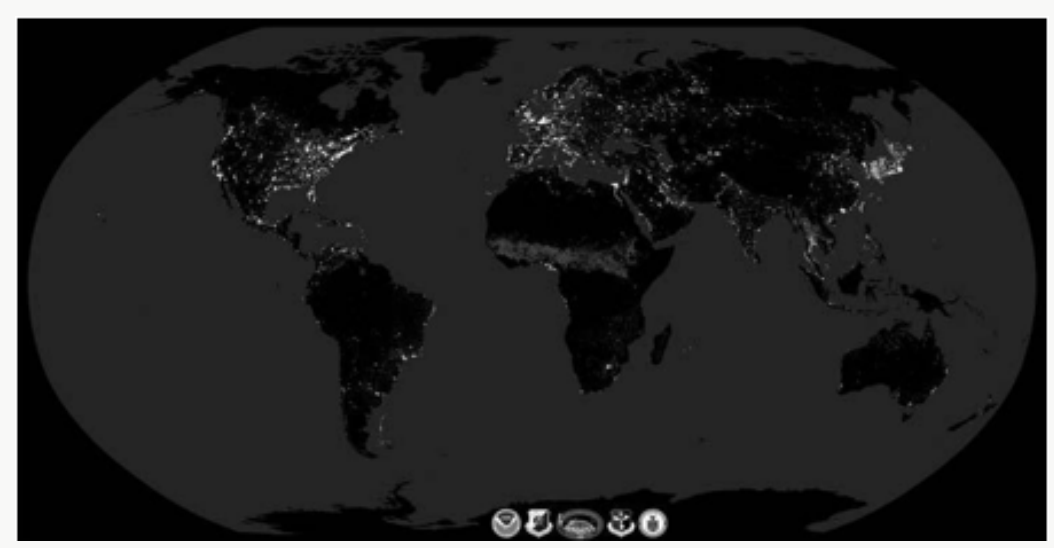

Figure 1: Elvidge \& M. L. Imhoff, 2001.

Figure 1 Distribution of artificial lights visible from space. Produced using cloud-free portions of low-light imaging data acquired by the U.S. Air Force Defense Meteorological Satellite Program (DMSP) Operational Linescan System (OLS). Four types of lights are included: human settlements (cities, towns, and villages), fires (defined as ephemeral lights on land), gas flares, and heavily lit fishing boats. See Elvidge [3] for details. Image, data processing, and descriptive text by the National Oceanic and Atmospheric Administration's National Geophysical Data Center.

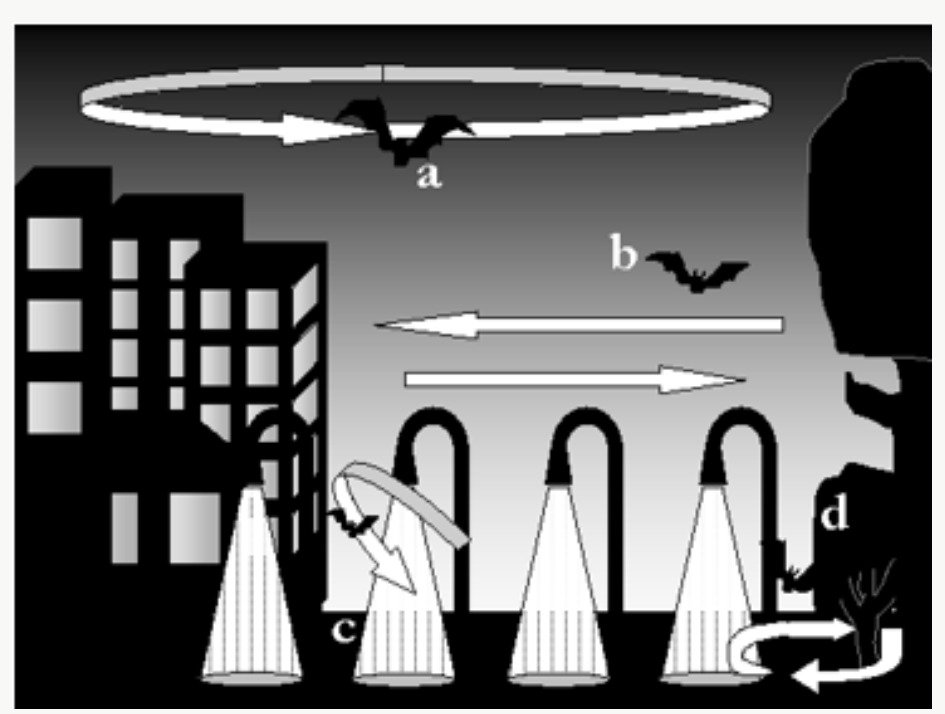

Figure 2: Unintentional birds' moving across streetlights designed by smart lighting (Elvidge \& M. L. Imhoff, 2001). 
As Figure 2 represents, smart lighting system makes wrong diagnosis, under the influence of birds or insects' motions, illuminating by no reason. This, which is one disadvantage, causes costs, energy, and capital losses unlike this research objective i.e. energy saving (economic project). Thus, the system requires be designed in a way to identify the problems and react to the problems. As Figure 3 shows, captivity effect system can remove the insects before approaching to the lights. This system emits some waves, creates overlap among lights, and prevents insects' infiltration. Moreover, using sound waves can be effective so that its frequency is not much problematic to human and at the same time inhibits birds' approaching to the project site.

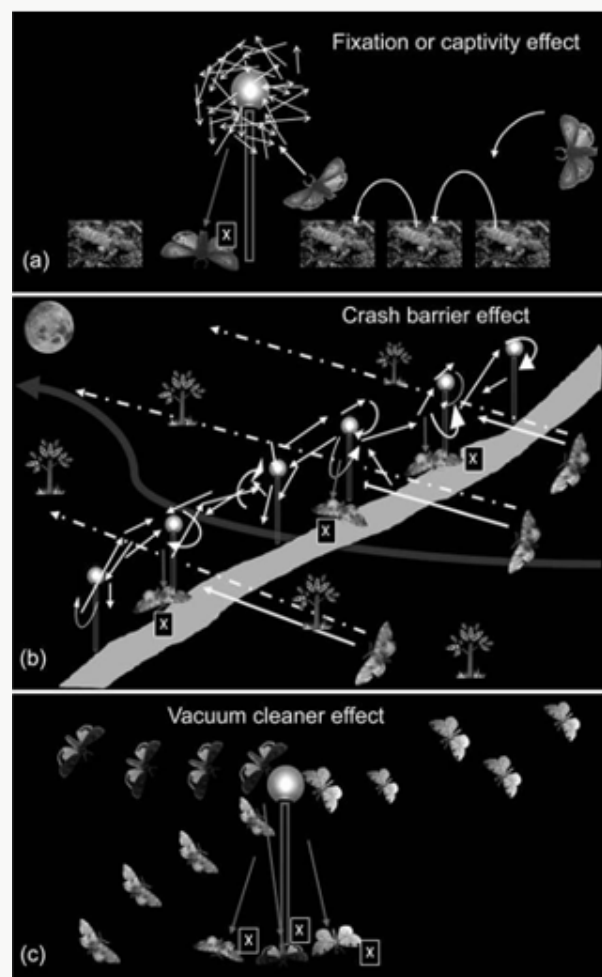

Figure 3: Using captivity effect system in order to inhibit insects' approaching (Elvidge \& M. L. Imhoff, 2001).

Companies and manufacturers made some efforts to improve the system performance and achieved some success; though, lacking perfect and comprehensive results, a promising future with no problem anticipated. In spite of knowing motion detection technology for construction environments, implementing it in outdoors and streets encounters us with complex problems such as stray animals, trees, wind, and climate changing. Recently, a new product naming Tvilight designed for solving the current problems. This technology includes eight sensors distinguishing people and vehicles from other environmental factors. Detection data communicates less than a hundredth of a second among different posts allowing them to properly react by estimating vehicle's speed and traffic line.

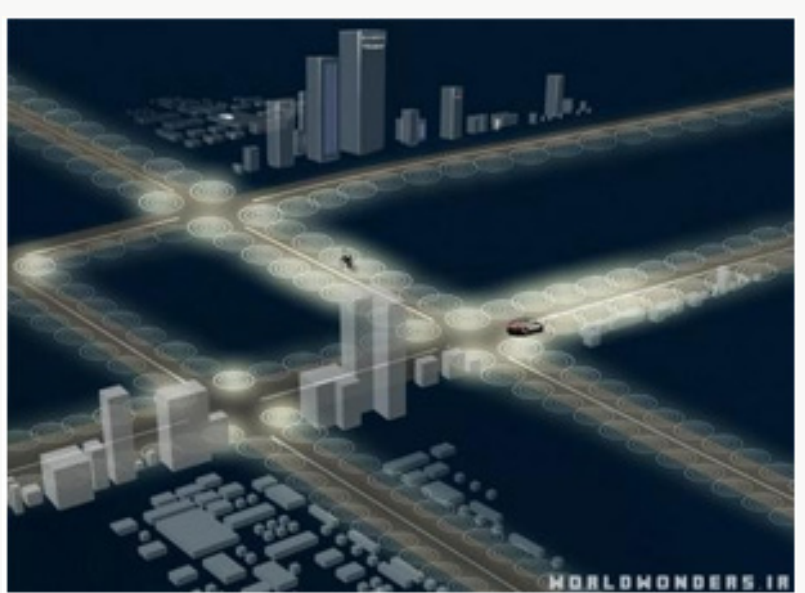

Figure 4: Illuminating of lighting system in human and veheicles' motion range. 
As seen in Figure 4, any sensor determines pedestrian or vehicle motion range by measuring speed and controls lighting of any identified range. The product options make lights' lighting reduced, namely in rural roads (roads outside city) and at particular times, up to $30 \%$, or in mostly empty areas such as parking lots up to $70 \%$.
These are surprising. Cities, states, and in general, counties can save up to $50 \%$ of lights' repairing and maintain costs and up to $80 \%$ in energy usage for street lighting, as needed. $40 \%$ of state's energy costs in Europe dedicated to street lighting; thus, using these lights and cost reduction can have incredible effects.

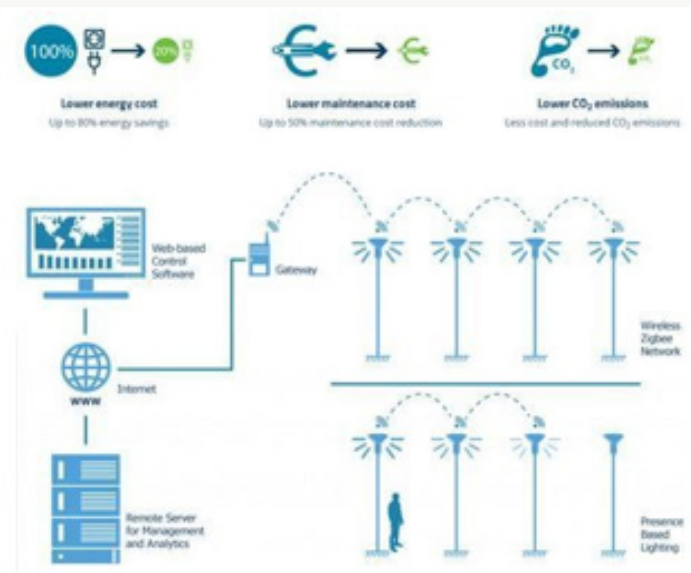

Figure 5: System structure and function.

Figure 5 illustrates how this system operates, the information transfers to the central communication system, which is globally controllable. Then, the obtained data estimated and turning on order issued for the assigned lights through wireless system. It also recommends some energy saving points so that energy consumption reduced to $20 \%$. The system manufacturer engineers largely maneuvered on the product dynamic so that it stated that it could be implemented on all streets and lights whether traditional or modern. The sensors can add to the old, existed lights in roads and or LED lamps. Now, it is applied in Holland an Ireland; it is also developing in Germany, Canada, and USA (http://7ww.ir/1467) [4]. One of the key elements of Smart City is a management system and street lighting control system. It allows intelligent management of individual lamps, can reduce energy consumption by dimming lighting in hours with little traffic, and areas of lesser importance. The use of this type of technology, reduce management and maintenance costs and reduces the cost of electricity consumption by up to $40 \%$. Intelligent lighting network can provide the basis for intelligent power distribution in urban areas. Typically, an intelligent lighting management system is the extension of the existing conventional lighting system. Implementation involves installing controllers in each lamp. Controller communicate with the control server via the existing power grid using Lon Works protocol and technology, PLC (Power Line Communication). Control server securely communicates through a network to the central management and control system. The transmission technology which is used based on 3G, Wi-Fi and Wi-MAX.

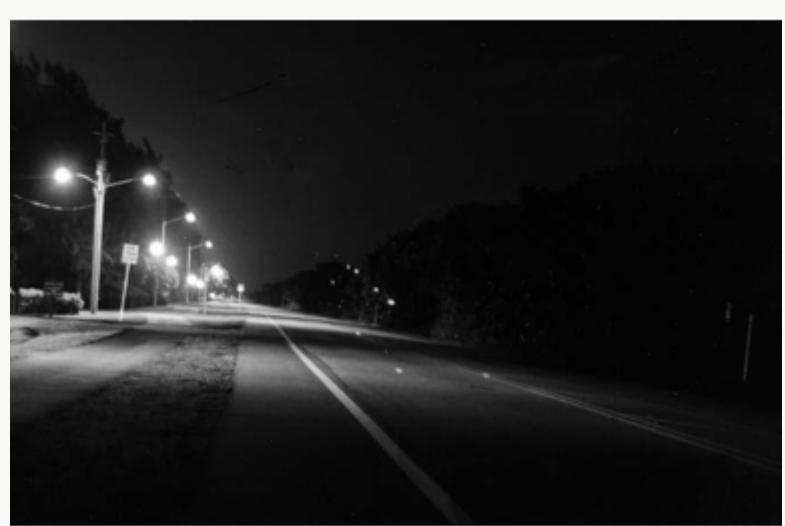

Figure 6: Traditional street lighting (Elvidge \& M. L. Imhoff, 2001).

Central management system allows full control of all lamps covered by surveillance. Allowing to configure different parameters depending on scenarios e.g. on / off, dimming lights in which you want to start saving. It provides information about the current operation of infrastructure, failures and reports of malfunctioning lamps. The graphically illustrates the operation of the system and presents reports on energy consumption. Smart lighting management system can be extended with additional elements 
for the implementation of advanced features. Such elements may include illumination sensors, motion detectors and sensors reporting environmental data (http://www.greeneris.com/) [5]. Indeed, as seen in Figure 6, traditionally using of lighting systems not only wastes the capitals and resources, but also it has no diversity, difference, and beauty. This system presents a straightforward, unchangeable appearance depriving the cities of dynamic, diversity, and visual beauty (Figure 6).

Applying a digital or radiation imaging system, if possible, better detects the passing object; then, avoids many problems such as wrong detection. The sensors only activate whenever human and vehicles pass preventing wrong lighting (Figure 7). More than a century after the introduction of incandescent lighting and half a century after the introduction of fluorescent lighting, solid- state light sources are revolutionizing an increasing number of applications. Whereas the efficiency of conventional incandescent and fluorescent lights is limited by fundamental factors that cannot be overcome, the efficiency of solid-state sources is limited only by human creativity and imagination. The high efficiency of solidstate sources already provides energy savings and environmental benefits in a number of applications. However, solid-state sources also offer controllability of their spectral power distribution, spatial distribution, color temperature, temporal modulation, and polarization properties. Such "smart" light sources can adjust to specific environments and requirements, a property that could result in tremendous benefits in lighting, automobiles, transportation, communication, imaging, agriculture, and medicine. Fred Schubert and Kyu Kim [6].
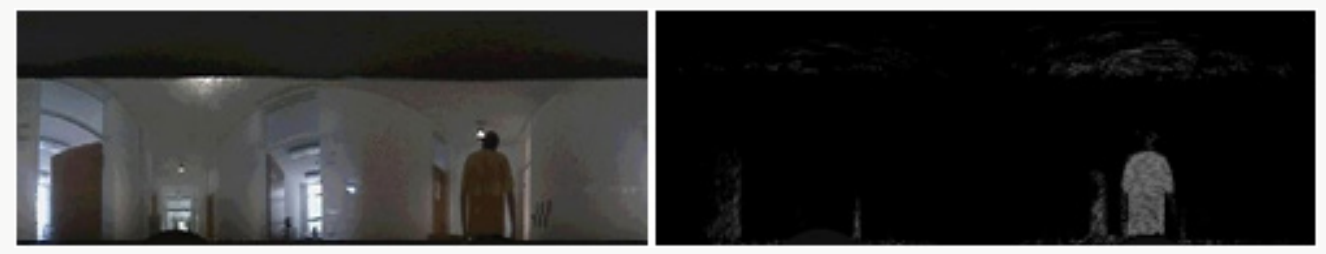

Figure 7: (Illmann, Kluge, \& Prassler).

\section{Smart Lighting}

In addition to the energy savings and positive environmental effects promised by solid-state lighting, solid-state sources in particular, LED-based sources offer what was inconceivable with conventional sources: controllability of their spectral, spatial, temporal, and polarization properties as well as their color temperature. Technologies currently emerging are expected to enable tremendous benefits in lighting, automobiles, transportation, communication, imaging, agriculture, and medicine. Fred Schubert and Kyu Kim [6].

Another potential benefit of smart lighting originates in the ability to rapidly modulate the output power of LED-based light sources, thereby enabling communication features. New modes of communication based on room-light sources would help to reduce the overcrowding of the radio frequency bands. Of course, the visual appearance of such communicative light sources would be indistinguishable from conventional sources. In automotive communication applications, brake lights could communicate an emergency braking maneuver to a following car. Headlights could inform a red traffic light of an approaching car while fully maintaining their normal function as headlights. Smart road signs could flash warnings specifically to drivers that approach a dangerous curve with excessive speed. Room lights could broadcast messages, alarms, and other types of information, without any noticeable change in the illumination quality. Modulation rates in the megahertz range are possible, with the limiting factor being the device resistance and capacitance (RC time) of high-power devices. The large-area junction capacitance means that such devices would be limited by RC time rather than by spontaneous lifetime. Fred Schubert and Kyu Kim [6].
The implemented process must be economic in long-term in addition to its economic justification at the beginning of the project; the spent investments and costs must return in short-term in order to develop country through energy and financial saving. Economical risk factors in long-term include selecting improper and problematic materials. For instance, selecting inappropriate color and light type may cause insects' attraction, which harms the lights and urban space and or leads to chemical effects and serious damages on colored surfaces Bekrani and Mahdizade Seraj, fall and winter [7]. However, lighting sources with larger thermal effects may be useful against humidity and proliferation of some types of moss or microorganisms, can also lead to harmful moisture deformation, intensified airflow, and excess growing of some plants (www.aftabir.com) [8].

\section{Features}

Comfortable lighting with dimming for scenes: Appropriate lighting and scene control based on specific environment or condition can not only provide better living situation, but also save energy consumption. You can enjoy a pleasant lighting environment by simply pressing a button. Smart lighting with body detection: According to surveys, most lamps in offices without a worker are lighted over $70 \%$ of the office hours. The LED drivers of DDL series with built-in PIR detectors can automatically execute continuous illumination adjustment. Thus, secure, comfortable, and energysaving smart lighting control can be achieved. Major parameters such as illumination and delay time can be varied on demand. There are two setting modes to be used in smart lighting control. As you can see in Figure 8

a) Single mode: the output power of every LED driver in the DDL series is determined by the individual PIR detector. 

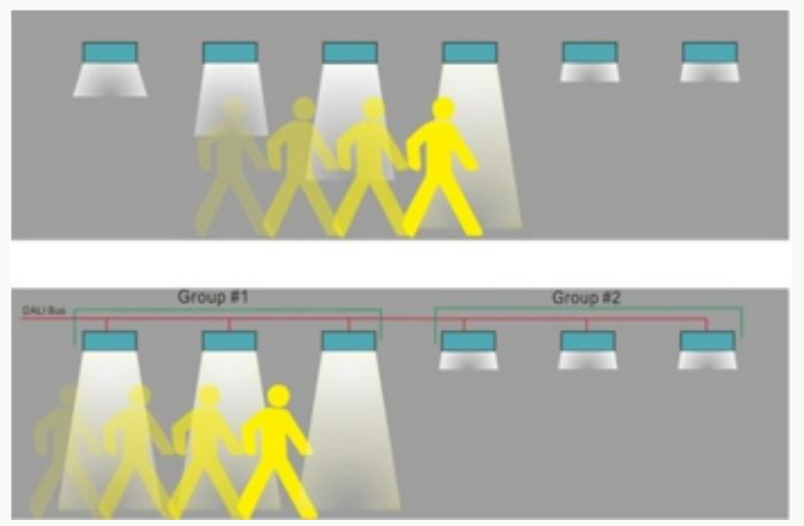

Figure 8: Classification of lights.

b) Group mode: every LED driver in the DDL series can share the outcome of other PIR detectors and offer the information to other lamps within the same group. Thus, all lamps in a group can be turned on simultaneously.

Complete Control for Systems or Individuals: The LED drivers of DDL series with built-in DALI interface and PIR detectors can create the simplest smart lighting control system to date. Either a lighting network in a building or an individual lamp can be completely controlled by our products. Automatic energysaving with schedule control: By using the scheduling function, on/off or illumination of lamps at specified time can be controlled based on your schedule. Hence, target of energy conservation can be achieved automatically. Both requirements of "limited budget" and "uncompromising efficiency" can be satisfied concurrently. The LED drivers of DDL series can provide more (http://www. ledinside.com/) [9].

\section{Specifications}
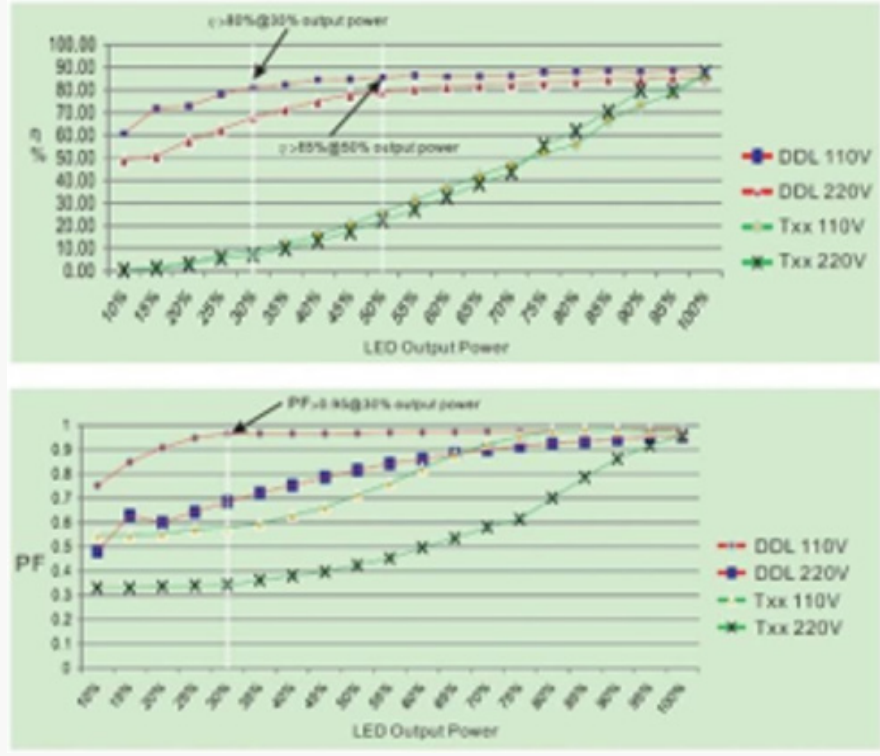

Figure 9

Figure 9 shows the power of external smart lights and studies the lights' differences.

\section{Conclusion}

Figure 10 (http://www.ledinside.com/) [9].

The primary objective of human being in utilizing the technology is service delivery. Smart lighting must consider human respect as its primary seeking for comfort and convenience in all areas of the community including economic and aesthetic-oriented diversity
[10-20]. Obtained results of the present study and other studies indicate that if required infrastructures and credits provided for such project and implemented based on standards using the least disadvantage, then it will have wonderful economic gain and yield in long-term. It not only develops the economy, but also creates the sense of beauty and diversity, and in an advanced sense, it teaches the culture of saving. This lighting model, regarding its highadvanced technology, is used in traditional cities if it has low error and high detection ability. Moreover, it leads to economic prosperity and pave the road to development road. 


\begin{tabular}{|c|c|c|c|}
\hline TYPE: & Input Power & $\begin{array}{l}\text { Operating } \\
\text { Temperature }\end{array}$ & LED Load \\
\hline $\begin{array}{l}\text { DDL-30-24-CB } \\
\text { DDL-30-24-CE }\end{array}$ & $\begin{array}{l}\mathrm{AC} 100-240 \mathrm{~V} \\
50.60 \mathrm{~Hz}\end{array}$ & $0-70 \mathrm{C}$ & $30 \mathrm{~W}(24 \mathrm{~V} / 1.25 \mathrm{~A})$ \\
\hline $\begin{array}{l}\text { DDL-30-27-CB } \\
\text { DDL-30-27-CE }\end{array}$ & $\begin{array}{l}\mathrm{AC} 100-240 \mathrm{~V} \\
50.60 \mathrm{~Hz}\end{array}$ & $0-70^{\circ} \mathrm{C}$ & $30 \mathrm{~W}(27 \mathrm{~V} / 1.12 \mathrm{~A})$ \\
\hline $\begin{array}{l}\text { DDL-30-36-CB } \\
\text { DDL-30-36-CE }\end{array}$ & $\begin{array}{l}\mathrm{AC} 100-240 \mathrm{~V} \\
50 \mathrm{O} 6 \mathrm{~Hz}\end{array}$ & $0-70 \mathrm{C}$ & $30 \mathrm{~W}(36 \mathrm{~V} / 0.84 \mathrm{~A})$ \\
\hline $\begin{array}{l}\text { DDL-30-40-CB } \\
\text { DDL-30-40-CE }\end{array}$ & $\begin{array}{l}\mathrm{AC} 100-240 \mathrm{~V} \\
50.60 \mathrm{~Hz}\end{array}$ & $0-700^{\circ}$ & $30 \mathrm{~W}(40 \mathrm{~V} / 0.75 \mathrm{~A})$ \\
\hline $\begin{array}{l}\text { DDL-30-48-CB } \\
\text { DDL-30-48-CE }\end{array}$ & $\begin{array}{l}\mathrm{AC} 100-240 \mathrm{~V} \\
50.60 \mathrm{~Hz}\end{array}$ & $0-70^{\circ} \mathrm{C}$ & $30 \mathrm{~W}(48 \mathrm{~V} / 0.63 \mathrm{~A})$ \\
\hline DDL-45-24-CB & $\begin{array}{l}\text { AC100 }-240 \mathrm{~V} \\
50.60 \mathrm{~Hz}\end{array}$ & $0-70^{\circ} \mathrm{C}$ & $45 \mathrm{~W}(24 \mathrm{~V} / 1.88 \mathrm{~A})$ \\
\hline DDL-45-27-CB & $\begin{array}{l}\mathrm{AC} 100-240 \mathrm{~V} \\
50.60 \mathrm{~Hz}\end{array}$ & $0-700^{\circ}$ & $45 \mathrm{~W}(27 \mathrm{~V} / 1.67 \mathrm{~A})$ \\
\hline DDL-45-36-CB & $\begin{array}{l}\mathrm{AC} 100-240 \mathrm{~V} \\
50.60 \mathrm{~Hz}\end{array}$ & $0-70 \mathrm{C}$ & $45 \mathrm{~W}(36 \mathrm{~V} / 1.25 \mathrm{~A})$ \\
\hline DDL $-45-40-C B$ & $\begin{array}{l}\mathrm{AC} 100-240 \mathrm{~V} \\
50.60 \mathrm{~Hz}\end{array}$ & $0-70 \mathrm{C}$ & $45 \mathrm{~W}(40 \mathrm{~V} / 1.13 \mathrm{~A})$ \\
\hline DDL-45-48-CB & $\begin{array}{l}\mathrm{AC} 100-240 \mathrm{~V} \\
50.60 \mathrm{~Hz}\end{array}$ & $0-70^{\circ} \mathrm{C}$ & $45 \mathrm{~W}(48 \mathrm{~V}, 0.94 \mathrm{~A})$ \\
\hline
\end{tabular}

Figure 10

\section{References}

1. Ghotbi Ravandi MR, Khanjani N, Nadri F, A Nadri, A Toolabi, et al. (2011) Evaluating lighting intensity and UV radiation in the libraries of Medical Sciences University. Journal of occupational health 8(4): 29-35.

2. Mahaleh AT, Kaveh (2008) An introduction to the basics of lighting design, Tehran: designer.

3. Elvidge CD, ML Imhoff, KE Baugh, VR Hobson, I Nelson, et al. (2001) Night-time lights of the world. ISPRS Journal of Photogrammetry \& Remote Sensing 56(2): 81-99.

4. http://7ww.ir/1467/

5. http://www.greeneris.com/

6. Fred Schubert E, Kyu Kim J (2005) Solid-State Light Sources Getting Smart. Science 308(5726): 1274-1278.

7. Bekrani M, Mahdizade Seraj F (2013) Lighting mechanism of ancient buildings in urban areas; case study: Sheikh Lotfollah. Journal Architecture and restoration in Iran 6: 99-110.

8. www.aftabir.com

9. http://www.ledinside.com/

10. Crowell B (2003) Electricity and Magnetism. Fullerton, California: Light and Matter, USA.
11. Cinzano P, Falchi F, Elvidge D (2001) The first world atlas of the artificial night sky brightness. Monthly Notices of the Royal Astronomical Society 328(3): 689-707.

12. http://www.everredtronics.com/smart.led.lighting.html

13. http://www.libelium.com/smart-lighting-sensor-solution-for-smartcities/

14.http://www.smartlighting.rpi.edu/

15. Illmann J, Kluge B, Prassler E (2001) People Tracking Using Omnidirectional Vision and Range Information. Research Institute for Applied Knowledge Processing Helmholtz's Ulm, Germany pp. ssss4571.

16. Makansi J (2007) Lights Out: The Electricity Crisis, the Global Economy, and What It Means to You. New Jersey: John Wiley \& Sons Inc Hoboken, USA.

17. Nii H, Sugimoto M, Inami M (2005) Smart Light-Ultra High-Speed Projector for Spatial Multiplexing Optical Transmission.

18. Patterson W (2007) Keeping the Lights On: Towards Sustainable Electricity. London: Earthscan: Chatham House, UK.

19. Rich C, Longcore T (2006) Ecological Consequences of Artificial Night Lighting. Washington: Island Press, USA.

20.Zhao Y, Ikeda T (2009) Smart Light responsive Materials: AzobenzeneContaining Polymers and Liquid Crystals. New Jersey: John Wiley \& Sons Inc, Hoboken, USA. 
This work is licensed under Creative Commons Attribution 4.0 License

To Submit Your Article Click Here: $\quad$ Submit Article

DOI: 10.32474/TCEIA.2018.02.000149

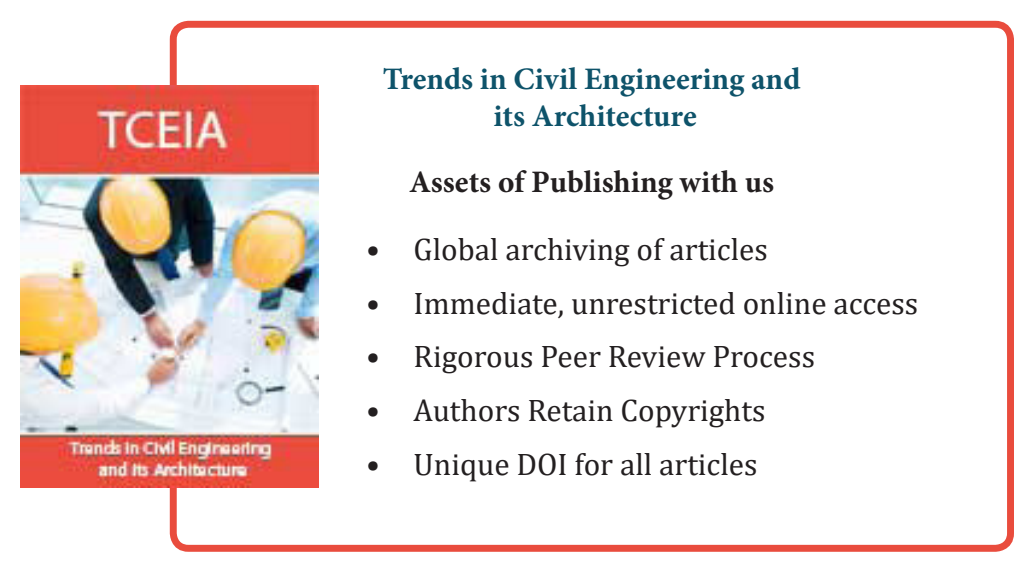

